\title{
Spectral function of the kondo model in high magnetic fields
}

Rosch, A.; Costi, T. A.; Paaske, Jens; Wolfle, P.

Published in:

Physical Review B Condensed Matter

DOI:

10.1103/PhysRevB.68.014430

Publication date:

2003

Document version

Early version, also known as pre-print

Citation for published version (APA):

Rosch, A., Costi, T. A., Paaske, J., \& Wolfle, P. (2003). Spectral function of the kondo model in high magnetic fields. Physical Review B Condensed Matter, 68(1), 014430. https://doi.org/10.1103/PhysRevB.68.014430 


\title{
Spectral function of the Kondo model in high magnetic fields
}

\author{
A. Rosch, T. A. Costi, J. Paaske, and P. Wölfle \\ Institut für Theorie der Kondensierten Materie, Universität Karlsruhe, D-76128 Karlsruhe, Germany
}

(Received 8 January 2003; published 29 July 2003)

\begin{abstract}
Using a recently developed perturbative renormalization group (RG) scheme, we calculate analytically the spectral function of a Kondo impurity for either large frequencies $\omega$ or large magnetic field $B$ and arbitrary frequencies. For large $\omega \gg \max \left[B, T_{K}\right]$ the spectral function decays as $1 / \ln ^{2}\left[\omega / T_{K}\right]$ with prefactors which depend on the magnetization. The spin-resolved spectral function displays a pronounced peak at $\omega \sim B$ with a characteristic asymmetry. In a detailed comparison with results from numerical renormalization group (NRG) and bare perturbation theory in next-to-leading logarithmic order, we show that our perturbative RG scheme is controlled by the small parameter $1 / \ln \left[\max (\omega, B) / T_{K}\right]$. Furthermore, we assess the ability of the NRG to resolve structures at finite frequencies.
\end{abstract}

DOI: 10.1103/PhysRevB.68.014430

PACS number(s): 75.20.Hr, 72.15.Qm, 73.63.Kv

\section{INTRODUCTION}

Only recently it has become possible to measure directly ${ }^{1-3}$ the so-called Kondo resonance, i.e., a sharp resonance characteristic for the spectral function of a single magnetic impurity in a metal. The Kondo resonance is probably the most direct manifestation of the Kondo effect ${ }^{4}$ : The antiferromagnetic coupling between conduction electrons and a localized spin causes a complex many-body resonance which ultimately screens the magnetic degree of freedom. The spectral function is directly proportional to a tunneling current, e.g., from the tip of a scanning tunneling microscope to a magnetic impurity on the surface of a metallic host. ${ }^{1}$ Alternatively it can be measured by tunneling through weak links into a quantum dot in the Kondo regime. ${ }^{2,3}$ This also opens the possibility of investigating the spectral function out of equilibrium. $^{3}$

While the spectral function is an interesting quantity in its own right, it also has many important applications. For example, it is directly related to the $T$ matrix, which describes the scattering of the conduction electrons (see below). It therefore determines not only the transport properties of magnetic impurities and quantum dots in the Kondo regime but it also plays an important role in controlling the distribution ${ }^{5}$ of electrons in metals contaminated by magnetic impurities. Furthermore, the spectral function of impurity models is central in determining the properties of lattice models within dynamical mean field theory. ${ }^{6}$

The spectral function of the Kondo model in a magnetic field has been calculated using a variety of techniques, ${ }^{4,7-15}$ including diagrammatics, mean-field treatments, and the quantum Monte Carlo technique. The most precise method, however, is the numerical renormalization group $^{16}$ (NRG) and its generalizations ${ }^{11,17-19}$ which allow the calculation of dynamical quantities. However, little is known analytically about the properties of the spectral function, particularly for high magnetic fields. For small $B$, the spectral function for small frequencies can be calculated from Fermi liquid theory and renormalized perturbation theory. ${ }^{13,14}$ We have recently developed $^{20}$ a perturbative renormalization group scheme based on frequency-dependent couplings for the Kondo model. While this method was formulated to describe the
Kondo effect out of equilibrium, it can also be used to calculate dynamical quantities in equilibrium in a controlled way for either large frequencies or large magnetic fields. We therefore use this method to determine analytically the spectral function in leading order of $1 / \ln \left[\max (B, \omega) / T_{K}\right]$, focusing our attention on equilibrium and vanishing temperature. This clarifies some long-standing questions on the asymptotics of the spectral function in the Kondo model. A detailed comparison with results from NRG and with perturbation theory allows us to check the assumptions underlying our perturbative RG approach.

\section{THE MODEL}

We consider the spectral function $\operatorname{Im} G_{\sigma}^{f}(\omega)$ of an electron in an impurity orbital within the Anderson model

$$
\begin{aligned}
H_{A}= & \sum_{\mathbf{k}, \sigma} \varepsilon_{\mathbf{k}} c_{\mathbf{k} \sigma}^{\dagger} c_{\mathbf{k} \sigma}+\sum_{\sigma} \epsilon_{f} f_{\sigma}^{\dagger} f_{\sigma}+V \sum_{\mathbf{k}, \sigma}\left(c_{\mathbf{k} \sigma}^{\dagger} f_{\sigma}+\text { H.c. }\right) \\
& +U n_{f, \uparrow} n_{f, \downarrow},
\end{aligned}
$$

where $c_{\mathbf{k} \sigma}^{\dagger}$ and $f_{\sigma}^{\dagger}$ are creation operators for electrons in the conduction band and in the impurity orbital, respectively, $n_{f \sigma}=f_{\sigma}^{\dagger} f_{\sigma}$, and $U$ is a large Coulomb interaction matrix element. We focus our attention on the Kondo regime, $\epsilon_{f} \ll$ $-V^{2} N_{F}, \epsilon_{f}+U \gg V^{2} N_{F}\left(N_{F}\right.$ being the conduction electron density of states), where the impurity orbital is occupied by a localized spin $\mathbf{S}$ (with $S=1 / 2$ ) and the problem can be mapped onto the Kondo Hamiltonian

$$
H_{K}=\sum_{\mathbf{k}, \sigma} \varepsilon_{\mathbf{k}} c_{\mathbf{k} \sigma}^{\dagger} c_{\mathbf{k} \sigma}-B S_{z}+J \sum_{\mathbf{k}, \mathbf{k}^{\prime}, \sigma, \sigma^{\prime}} \mathbf{S} \cdot c_{\mathbf{k}^{\prime} \sigma^{\prime}}^{\dagger} \boldsymbol{\tau}_{\sigma^{\prime} \sigma} c_{\mathbf{k} \sigma},
$$

where $\boldsymbol{\tau}$ is the vector of Pauli matrices. Note that we have coupled an extra magnetic field $B$ to the impurity, ${ }^{21}$ measuring $B$ in units of the Zeeman splitting.

The Green function $G_{\sigma}^{f}$ within the Anderson model is directly related to the $T$ matrix $T_{\sigma}(\omega)=V^{2} G_{\sigma}^{f}(\omega)$ of the conduction electrons, defined by

$$
g_{\sigma, \mathbf{k}, \mathbf{k}^{\prime}}^{c}(\omega)=g_{\mathbf{k}}^{0}(\omega) \delta\left(\mathbf{k}-\mathbf{k}^{\prime}\right)+g_{\mathbf{k}}^{0}(\omega) T_{\sigma}(\omega) g_{\mathbf{k}^{\prime}}^{0}(\omega),
$$


where $g_{\sigma, \mathbf{k}, \mathbf{k}^{\prime}}^{c}\left(g_{\mathbf{k}}^{0}\right)$ is the (bare) Green function of the conduction electrons. While it is not completely obvious how to define a spectral function for a Kondo impurity, the $T$ matrix of the Kondo model can easily be identified with the following correlation function, e.g., using equations of motion, ${ }^{10}$

$$
T_{\sigma}(\omega)=V^{2} G_{\sigma}^{f}(\omega)=J\left\langle S_{z}\right\rangle+J^{2}\left\langle\left\langle\mathbf{S} c_{\alpha}^{\dagger} \boldsymbol{\tau}_{\alpha \sigma} ; \mathbf{S} \boldsymbol{\tau}_{\sigma \alpha^{\prime}} c_{\alpha^{\prime}}\right\rangle\right\rangle,
$$

where $\langle\langle\cdots\rangle\rangle$ denotes a retarded correlation function. Formally, we will consider the usual "scaling limit," where the ratios of frequency or magnetic field and Kondo temperature, $\omega / T_{K}, B / T_{K}$, are kept fixed while the ratio of $T_{K}$ and all "high energy" scales like bandwidth $D_{0}$, level position, or interaction are sent to zero, $T_{K} / D_{0}, T_{K} / U, T_{K} / \epsilon_{f} \rightarrow 0$. In this limit, the dimensionless product of the $T$ matrix and the density of states, $N_{F} \operatorname{Im} T(\omega)$, is a universal function of $\omega / T_{K}$ and $B / T_{K}$ and it coincides with the low-frequency part of the spectral function $N_{F} V^{2} \operatorname{Im} G_{\sigma}^{f}(\omega)$ of the Anderson impurity. Therefore $N_{F} \operatorname{Im} T_{\sigma}(\omega)$ is the quantity to be studied below.

\section{PERTURBATIVE RG METHOD}

The perturbation theory of the Kondo model is characterized by logarithmic divergences. The method of choice for the resummation of leading logarithmic corrections is the perturbative renormalization group (RG). In Ref. 20 we have developed a certain formulation of perturbative RG based on frequency-dependent coupling constants. A thorough discussion of this approach will be published elsewhere, ${ }^{22}$ here we will use the RG equations without further derivation and check the results extensively in a comparison with bare perturbation theory and NRG.

The RG is formulated in terms of frequency- and spindependent vertices ${ }^{23} g g_{\gamma \omega_{f} ; \gamma^{\prime} \omega_{f}^{\prime}}^{\sigma \omega_{c} ; \sigma^{\prime} \omega^{\prime}}$ where $\sigma$ and $\omega_{c}\left(\gamma\right.$ and $\left.\omega_{f}\right)$ denote the spin and energy of the incoming electron (the incoming spin); see Ref. 20. Primed quantities refer to outgoing particles. Before renormalization starts, the vertex $g_{\gamma \omega_{f} ; \gamma^{\prime} \omega_{f}^{\prime}}^{\sigma \omega_{c} ; \sigma^{\prime} \omega_{c}^{\prime}}$ is just given by the bare coupling $N_{F} J \boldsymbol{\tau}_{\gamma \gamma^{\prime}} \boldsymbol{\tau}_{\sigma \sigma^{\prime}}$ independent of frequencies. In leading order of the relevant expansion parameter $1 / \ln \left[\max (\omega, B) / T_{K}\right]$, one can set the energy of the spin on-shell $\omega_{f}=-\gamma B / 2, \omega_{f}^{\prime}=-\gamma^{\prime} B / 2$ to keep track only of the energy of the incoming electron. The vertex is parametrized as

$$
\begin{aligned}
g_{\gamma,-\gamma B / 2 ; \gamma^{\prime},-\gamma^{\prime} B / 2}^{\sigma, \omega ; \sigma^{\prime}, \omega-\left(\gamma-\gamma^{\prime}\right) B / 2}= & \tau_{\gamma \gamma^{\prime}}^{z} \tau_{\sigma \sigma^{\prime}}^{z} \tilde{g}_{z \sigma}(\omega) \\
& +\left(\tau_{\gamma \gamma^{\prime}}^{x} \tau_{\sigma \sigma^{\prime}}^{x}+\tau_{\gamma \gamma^{\prime}}^{y} \tau_{\sigma \sigma^{\prime}}^{y}\right) \\
& \times \tilde{g}_{\perp}(\omega-\gamma B / 2) .
\end{aligned}
$$

The one-loop RG equations (see Fig. 1) read $^{20}$ in terms of the running cutoff $D$

$$
\frac{\partial \tilde{g}_{z \sigma}(\omega)}{\partial \ln D}=-2 \tilde{g}_{\perp}(B / 2)^{2} \Theta(D-|\omega+\sigma B|),
$$

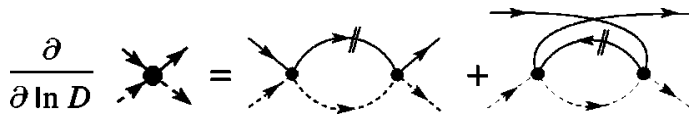

FIG. 1. Diagrammatic form of the RG equation (6). The strokes symbolize derivatives with respect to $\ln D$; see Ref. 20 for details.

$$
\frac{\partial \tilde{g}_{\perp}(\omega)}{\partial \ln D}=-\sum_{\sigma=-1,1} \tilde{g}_{\perp}\left(\frac{\sigma B}{2}\right) \tilde{g}_{z \sigma}(0) \Theta\left(D-\left|\omega+\frac{\sigma B}{2}\right|\right),
$$

with $\tilde{g}_{\perp}(\omega)=\tilde{g}_{\perp}(-\omega), \tilde{g}_{z \uparrow}(\omega)=\tilde{g}_{z \downarrow}(-\omega)$ and the initial conditions $\tilde{g}_{z \sigma}(\omega)=\tilde{g}_{\perp}(\omega)=J N_{F}$ at the bare cut-off $D_{0}$. The theta function $\Theta(\omega)$ describes that various virtual processes are not possible if the running cutoff $D$ gets too small. Note that one recovers the usual poor man's scaling equation ${ }^{24}$ if one considers only the limit $\omega \rightarrow 0$ as it is usually done in RG schemes.

The RG equations (6) are easily solved and we obtain for $D \rightarrow 0$

$$
\begin{aligned}
\tilde{g}_{z \sigma}(\omega)= & \Theta(|\omega+\sigma B|-B) \frac{1}{2 \ln \left[|\omega+\sigma B| / T_{K}\right]} \\
& +\Theta(B-|\omega+\sigma B|) \frac{1}{2 \ln \left[B / T_{K}\right]}\left|\frac{B}{\omega+\sigma B}\right|^{1 / \ln \left[B / T_{K}\right]},
\end{aligned}
$$

$$
\begin{aligned}
\tilde{g}_{\perp}(\omega)= & \sum_{\sigma} \Theta(|\omega+\sigma B / 2|-B) \frac{1}{4 \ln \left[|\omega+\sigma B / 2| / T_{K}\right]} \\
& +\Theta\left[B-\left|\omega+\sigma \frac{B}{2}\right|\right] \\
& \times\left(\frac{1}{2 \ln \left[B / T_{K}\right]}\left|\frac{B}{\omega+\sigma B / 2}\right|^{1 / 2 \ln \left[B / T_{K}\right]}-\frac{1}{4 \ln \left[B / T_{K}\right]}\right),
\end{aligned}
$$

where within our one-loop approach $T_{K}=D_{0} e^{-1 /\left(2 N_{F} J\right)}$. As expected, the dimensionless renormalized couplings are functions of $\omega / T_{K}$ and $B / T_{K}$ only.

It is important to realize that the logarithmic renormalizations resummed in Eq. (6) are finally cut off by the relaxation rate $\Gamma$ of the spin. As the relevant processes involve at least one spin flip, we identify $\Gamma$ with the transverse spin relaxation rate $1 / T_{2}$, which is given in terms of the renormalized couplings as ${ }^{20}$

$$
\begin{aligned}
\Gamma= & \frac{\pi}{4} \sum_{\gamma=-1,1} \int d \omega\left[\tilde{g}_{z \gamma}(\omega)^{2} f_{\omega}\left(1-f_{\omega}\right)\right. \\
& \left.+\tilde{g}_{\perp}(\omega-\gamma B / 2)^{2} f_{\omega}\left(1-f_{\omega-\gamma B}\right)\right],
\end{aligned}
$$

where $f_{\omega}$ is the Fermi function. The rate $\Gamma$ cuts off the power-law singularities at $\omega= \pm B$ (or $\omega= \pm B / 2$ ) and we implement this by replacing $B /|\omega+\sigma B|$ by $\sqrt{\Gamma^{2}+B^{2}} / \sqrt{\Gamma^{2}+(\omega+\sigma B)^{2}}$ in Eq. (7). The errors introduced by this heuristic replacement are small $^{22}$ in $1 / \ln \left[\max (B, \omega) / T_{K}\right]$. For $B \gg T_{K}$, we obtain $\Gamma$ $\approx \pi B /\left(16 \ln ^{2}\left[B / T_{K}\right]\right) \gg T_{K}$ and therefore $\tilde{g}_{z \sigma}(\omega) \ll 1$ and $\tilde{g}_{\perp}(\omega) \ll 1$. Accordingly, the perturbative RG is valid in this 


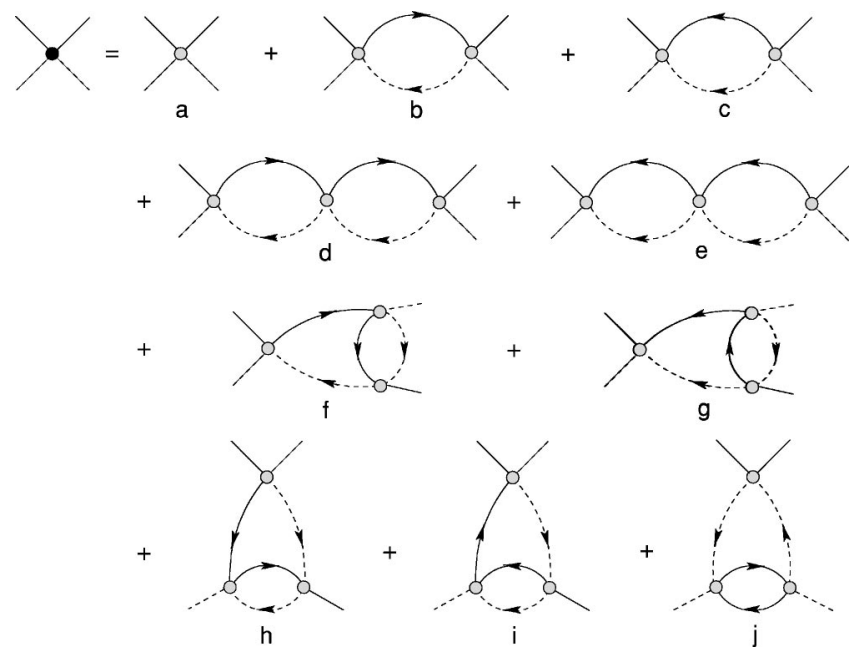

FIG. 2. Diagrams contributing to the vertex renormalization up to order $g^{3}$. Solid line: conduction electrons, dashed line: pseudofermions representing the spin. See Ref. 20 for the corresponding Feynman rules. The (nonparquet) diagram (j) does not contribute to order $g^{3} \ln ^{2} D_{0}$.

regime $^{25}$ for all frequencies (while for $B \lesssim T_{K}$ it can be used only for $\omega \gg T_{K}$ ). The smallness of the renormalized couplings for either large $B$ or large $\omega$ is the main reason why our calculation is controlled by $\left.1 / \ln [\max (\omega, B]) / T_{K}\right]$ as, e.g., higher-order corrections to Eq. (6) remain small.

To check the assumptions underlying our formulation of the perturbative RG for $\omega$-dependent vertices, we have calculated the vertices in next-to-leading logarithmic order in bare perturbation theory by evaluating the diagrams shown in Fig. 2. Such a test seems to be useful as RG schemes for $\omega$-dependent vertices are not used very often. Neglecting terms of order $g^{3} \ln D_{0}$, we obtain

$$
\begin{aligned}
\tilde{g}_{\perp}(\omega) \approx & g+g^{2}\left(\ln \frac{D_{0}}{|\omega-B / 2|}+\ln \frac{D_{0}}{|\omega+B / 2|}\right) \\
+ & \frac{g^{3}}{2}\left(\ln ^{2} \frac{D_{0}}{|\omega-B / 2|}+6 \ln \frac{D_{0}}{|\omega-B / 2|} \ln \frac{D_{0}}{|\omega+B / 2|}\right. \\
+ & \left.\ln ^{2} \frac{D_{0}}{|\omega+B / 2|}\right), \\
\tilde{g}_{z \sigma}(\omega) \approx & g+2 g^{2} \ln \frac{D_{0}}{|\omega+\sigma B|}+2 g^{3}\left(\operatorname { l n } \frac { D _ { 0 } } { | \omega | } \left[\ln \frac{D_{0}}{|\omega+\sigma B|}\right.\right. \\
& \left.\left.-\ln \frac{D_{0}}{B}\right]+\ln \frac{D_{0}}{|\omega+\sigma B|}\left[\ln \frac{D_{0}}{|\omega+\sigma B|}+\ln \frac{D_{0}}{B}\right]\right)
\end{aligned}
$$

where $g=N_{F} J$. The perturbative RG is expected to resum all leading logarithmic terms. Indeed, if we expand Eq. (7) carefully in $g$ using that $T_{K}=D_{0} e^{-1 /(2 g)}$, we obtain the same logarithmic divergences in all relevant limits, $\omega \rightarrow \pm B / 2$ or $\omega \rightarrow \pm B, \omega \rightarrow \pm \infty$, and $\omega \rightarrow 0$ [the singularity at $\omega=0$ in Eq. (10) cancels]. One assumption which has not been checked in the perturbative calculation shown above is that

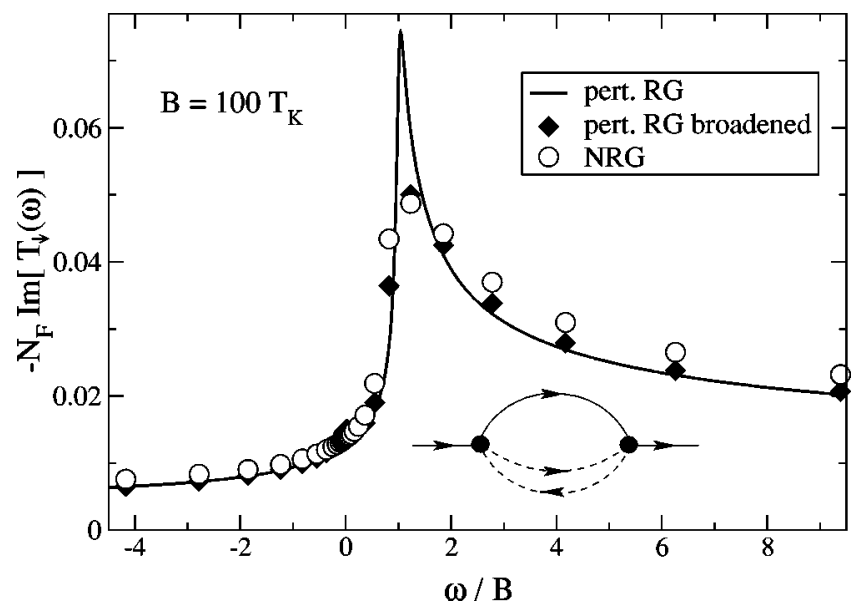

FIG. 3. $T$ matrix $-N_{F} \operatorname{Im}\left[T_{\downarrow}(\omega)\right]$ [or equivalently the spectral function $-N_{F} V^{2} \operatorname{Im} G_{\downarrow}^{f}(\omega)$ of an Anderson impurity] as a function of $\omega / B$ for $B=100 T_{K}$. Solid line: perturbative RG, open circles: NRG. The NRG is not able to resolve sharp peaks at high frequencies. For a direct comparison, we have therefore convoluted (filled diamonds) the result of the perturbative RG with the resolution function used in the NRG code. Inset: Diagram used to calculate the $T$ matrix (4) in leading order using renormalized vertices.

the logarithms are cut off by the spin-relaxation rate. To analyze this effect one would have to include self-energy insertions and corresponding vertex corrections as will be discussed in a future presentation ${ }^{22}$.

To leading order in $1 / \ln \left[\max (\omega, B) / T_{K}\right]$ the spectral function can be calculated by replacing the bare coupling constants in the lowest order expression (inset of Fig. 3) with the renormalized vertex (5) and one obtains ${ }^{26}$

$$
\begin{aligned}
N_{F} \operatorname{Im}\left[T_{\sigma}(\omega)\right]= & -\frac{\pi}{4}\left\{\tilde{g}_{z \sigma}(\omega)^{2}+2 \tilde{g}_{\perp}(\omega+\sigma B / 2)^{2}\right. \\
& \left.\times\left[1+\sigma M\left(2 f_{\Gamma}(\omega+\sigma B)-1\right)\right]\right\},
\end{aligned}
$$

where $M=2\left\langle S_{z}\right\rangle$ is the magnetization of the impurity normalized to 1 . The Fermi function $f_{\Gamma}(\omega \pm B)$ has to be broadened by $\Gamma, f_{\Gamma}(\omega)=1 / 2-\arctan [\omega / \Gamma] / \pi$. Diagrammatically this arises as the corresponding term in $T(\omega)$ contains a convolution with the spin-flip susceptibility $\chi^{+-}(\omega)$ (see inset of Fig. 3) and we used the fact that the $\omega$ dependence of the vertex $\tilde{g}_{\perp}(\omega)$ is sufficiently weak.

For $B \gg T_{K}$ one can replace $M$ in (11) by 1 in leading order of $1 / \ln \left[B / T_{K}\right]$. However, to obtain the correct large- $\omega$ behavior for arbitrary $B$ it is essential to use the exact local magnetization. For $B \sim T_{K}$ it is not possible to calculate $M$ within our RG scheme, and we therefore use the magnetization obtained from the Bethe ansatz (or the practically identical values from the NRG scheme).

Friedel's sum rule ${ }^{27}$ relates the spectral function at $\omega=0$ exactly to the local magnetization $N_{F} \operatorname{Im}\left[T_{\sigma}(\omega=0)\right]=$ $-1 / \pi \sin ^{2} \delta_{\sigma}$ where $\delta_{\sigma} / \pi=(1+\sigma M) / 2$. As a further check of our approach, we compare this result to our perturbative RG method. From Eqs. (7) and (11), we obtain asymptoti- 


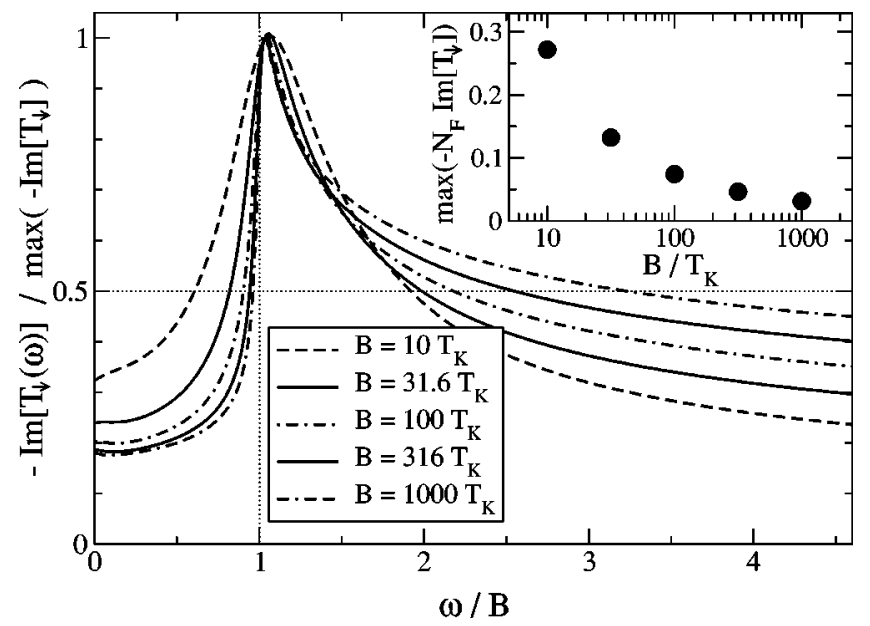

FIG. 4. Line shape of the spectral function as a function of $\omega / B$ calculated within perturbative RG. All curves are rescaled by their maximum. For increasing $B$ the peaks get more and more asymmetric. Note that a small systematic shift of the peak position towards lower frequencies for smaller $B$ is not included (see text). Inset: peak height as a function of $B / T_{K}$.

cally $N_{F} \operatorname{Im}\left[T_{\sigma}(\omega=0)\right] \approx-(\pi / 16) 1 / \ln ^{2}\left[B / T_{K}\right]$ for $B \gg T_{K}$, which is consistent with the asymptotic Bethe ansatz ${ }^{28}$ result $M=1-1 /\left(2 \ln \left[B / T_{K}\right]\right)-\cdots$.

A typical spectral function for a spin-down electron is shown in Fig. 3; the corresponding spin-up spectral function can be obtained from $\operatorname{Im} T_{\uparrow}(\omega)=\operatorname{Im} T_{\downarrow}(-\omega)$. For large frequencies the spectral function decays as

$$
N_{F} \operatorname{Im}\left[T_{\sigma}(\omega)\right] \approx-\pi \frac{3 \mp 2 \sigma M}{16 \ln ^{2}\left[\omega / T_{K}\right]} \text { for } \omega \rightarrow \pm \infty
$$

A similar result, discussed below, has been obtained by Logan and Dickens. ${ }^{13,29}$

For $\omega \sim B$ the spectral function is characterized by a pronounced and highly asymmetric peak. The width of the left flank is determined by $\Gamma$ and is therefore of order $B / \ln ^{2}\left[B / T_{K}\right]$. As $\Gamma / B \sim 1 / \ln ^{2}\left[B / T_{K}\right]$ decreases for increasing $B$, the left flank sharpens with increasing $B$ (see Fig. 4). As the width of the right flank increases for large $B$ the peak gets more and more asymmetric. Formally, the line shape of the right flank is characterized by a power law for $\Gamma \ll \omega-B$ $\ll B$; see Eq. (7). However, the corresponding exponents $\alpha$ of order $1 / \ln \left[B / T_{K}\right]$ are so small that it is not even in principle possible to extract the power-law behavior ${ }^{30}$ as $\alpha \ln [B / \Gamma]$ $\sim \ln \left[\ln \left(B / T_{K}\right)\right] / \ln \left(B / T_{K}\right) \ll 1$.

Within our perturbative RG method, the peak in the spectral function is always positioned at $\omega=B$; see Fig. 4. This is the correct $B \rightarrow \infty$ result. A subleading shift towards lower values is not included in our scheme which neglects renormalization of the real part of the self-energy. ${ }^{31}$ An estimate ${ }^{22}$ suggests that this effect is of order $B / \ln \left[B / T_{K}\right]$, consistent with results from Moore and Wen ${ }^{12}$ discussed below. For $B$ $\ll T_{K}$ it has been shown ${ }^{13,14,32}$ that the peak in the spectral function is positioned at $2 \mathrm{~B} / 3$. A splitting in the total spectral function $\operatorname{Im} T_{\uparrow}(\omega)+\operatorname{Im} T_{\downarrow}(\omega)$ can be observed ${ }^{10}$ for $B$ $\gtrsim 0.5 T_{K}^{*}$, where $T_{K}^{*} \approx 2.8 T_{K}$ is the half width at half maximum (HWHM) of the Kondo resonance at $T=0$.

\section{NRG METHOD}

The results of the perturbative RG approach to the equilibrium $T$ matrix $T(\omega)$ can be compared to numerical renormalization group (NRG) results for the same quantity. ${ }^{10}$ The NRG procedure ${ }^{16}$ consists of the following steps: (i) a logarithmic mesh of $\mathrm{k}$ points $k_{n}=\Lambda^{-n}$ is introduced about the Fermi wave vector $k_{F}=0$, and (ii) a unitary transformation of the $c_{k \sigma}$ is performed such that $f_{0 \sigma}=\Sigma_{k} c_{k \sigma}$ is the first operator in a new basis, $f_{n \sigma}, n=0,1, \ldots$, which tridiagonalizes $H_{c}=\Sigma_{k \sigma} \epsilon_{k \sigma} c_{k \sigma}^{\dagger} c_{k \sigma}$ in $k$ space, i.e., $H_{c}$ $\rightarrow \sum_{\sigma} \sum_{n=0}^{\infty} \Lambda^{-n / 2}\left(f_{n+1 \sigma}^{\dagger} f_{n \sigma}+\right.$ H.c. $)$. The Hamiltonian (2) with the above discretized form of the kinetic energy is now iteratively diagonalized by defining a sequence of finite size Hamiltonians $H_{N}=\sum_{\sigma, n=0}^{N-1} \Lambda^{-n / 2}\left(f_{n+1 \sigma}^{\dagger} f_{n \sigma}+\right.$ H.c. $)$ $+J \sum_{\sigma, \sigma^{\prime}} \mathbf{S} \cdot f_{0, \sigma^{\prime}}^{\dagger} \boldsymbol{\tau}_{\sigma^{\prime} \sigma} f_{0, \sigma}-B S_{z}$ for $N \geqslant 0$. For each $N$, this yields the excitation energies $E_{\lambda}^{N}$ and many-body eigenstates $|\lambda\rangle_{N}$ of $H_{N}$ at a corresponding set of energy scales $\omega_{N}$ defined by the smallest scale in $H_{N}, \omega_{N}=\Lambda^{-(N-1) / 2}$. Since the number of states grows as $4^{N}$, for $N>6$ only the lowest 500 or so states are retained for $H_{N}$. This limits the width of the spectrum of $H_{N}$ to $0 \leqslant \omega \leqslant K(\Lambda) \omega_{N}$, where $K(\Lambda) \approx 5$ for the value $\Lambda=1.5$ used in this paper. We are interested in $\operatorname{Im} T(\omega)=\operatorname{Im}\left\langle\left\langle O ; O^{\dagger}\right\rangle\right\rangle_{\rho}$, where $O=\mathbf{S} c_{\alpha}^{\dagger} \boldsymbol{\tau}_{\alpha \sigma}$ is defined in Eq. (4)and the subscript $\rho$ indicates the density matrix used to evaluate the thermodynamic averages (see below). The matrix elements $\langle m|O| n\rangle_{N}$, which are required for $T(\omega)$, are also calculated iteratively. $\operatorname{Im} T(\omega)$ is then constructed at a characteristic set of frequencies $\omega=\Omega_{N}$ from $H_{N}$ for each $N=0,1, \ldots$ via its Lehmann representation. This necessarily yields $\operatorname{Im} T(\omega)$ in the form of a set of weighted delta functions $\delta\left(\Omega_{N} \pm E_{\lambda}^{N}\right)$. Continuous spectra are obtained by replacing the delta functions by Gaussians of width $\eta_{N}: \delta\left(\Omega_{N} \pm E_{\lambda}^{N}\right) \rightarrow\left(1 / \eta_{N} \sqrt{\pi}\right) \exp \left[-\left(\Omega_{N} \pm E_{\lambda}^{N}\right)^{2} / \eta_{N}^{2}\right]$. As noted above, the spectrum of $H_{N}$ is known in a finite window of size $\approx 5 \omega_{N}$ for $\Lambda=1.5$. We therefore choose $\Omega_{N}=1.8 \omega_{N}$, not too low since the lower part of the spectrum is refined in later iterations and not too close to the upper bound of the spectrum which could be subject to truncation errors. We also set $\eta_{N}=0.39 \Omega_{N}$ of order the level structure of $H_{N}$. The main interest in this paper is to compare the NRG results for $\operatorname{Im} T(\omega, B, T=0)$ with the perturbative RG calculations in the limit $B \gg T_{K}$ where the latter are expected to be accurate. In this limit, the spectral features in $\operatorname{Im} T(\omega, B, T=0)$ lie at high frequencies $\omega \approx B$. A description of this finite-field, high-frequency part of the spectrum within NRG requires the use of a reduced density matrix ${ }^{11,32}$ calculated from the true ground state, i.e., $H_{N \rightarrow \infty}$, in place of the grand-canonical density matrix obtained from $H_{N}$. The necessity to use ground-state properties to calculate highfrequency features is, for example, evident from Eq. (12): the magnetization determines the large- $\omega$ tails. Nevertheless, the two approaches have been shown to give almost identical results at $B=0$ and to be in good agreement ${ }^{32}$ for fields $B$ $\lesssim 10 T_{K}$. 


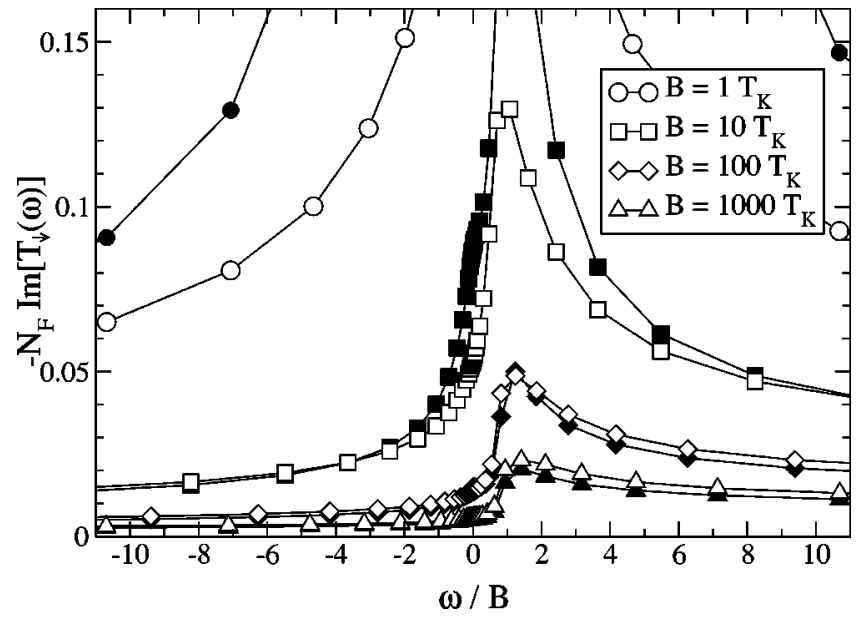

FIG. 5. Comparison of NRG (open symbols) and perturbative RG (filled symbols) results broadened by the NRG-resolution function (see caption of Fig. 3) for large magnetic fields, $B$ $=1,10,100,1000 T_{K}$.

\section{COMPARISONS}

How well does the perturbative RG method reproduce the spectral functions calculated within the NRG method? Figure 3 shows excellent agreement ${ }^{33}$ for $B / T_{K}=100$ both for $\omega$ $\rightarrow 0$ and $\omega \rightarrow \pm \infty$. However, large deviations arise for $\omega$ $\approx B$ where the peak within the perturbative $\mathrm{RG}$ method is much sharper and higher. This disagreement arises because the logarithmic discretization in energy used within the NRG method cannot resolve sharp features at high frequencies. More precisely, for a feature at a given frequency $\Omega_{0}$ with intrinsic width $\Delta_{0}$ it will fail to resolve it ${ }^{34}$ if $\Omega_{0}>\Delta_{0}$ since the broadening used at frequency $\Omega_{0}$ is necessarily of order $\Omega_{0}\left(0.39 \Omega_{0}\right.$ in our calculations $\left.{ }^{35}\right)$. This is the case here, since the spectral feature at $\Omega_{0}=B$ has a width on its left flank given by $\Delta_{0} \sim B / \ln ^{2}\left[B / T_{K}\right]$. Consequently, the width of this feature will not be captured by the NRG method for $B$ $\gg T_{K}$. To allow a direct comparison of NRG and perturbative RG methods, we have broadened the latter at the energy $\omega$ by convolution with the resolution function used in the NRG code, i.e., by a Gaussian of width $0.39 \omega$. After this broadening, the agreement of perturbative $\mathrm{RG}$ and $\mathrm{NRG}$ methods is excellent for all frequencies as long as $B \gg T_{K}$, see Figs. 3 and 5. For smaller $B$, the spectral function cannot be calculated reliably for small $\omega$ within our perturbative RG and indeed strong deviations can be seen in Fig. 5 for $B$ $\lesssim 10 T_{K}$. Figure 6 shows that the large $\omega$ behavior is nevertheless well reproduced which firmly establishes Eq. (12). Note that we replaced $M$ in Eq. (11) by the true ground-state magnetization for this comparison as discussed above.

We have argued that the small parameter controlling the validity of our perturbative $\mathrm{RG}$ method is $1 / \ln \left[\max (B, \omega) / T_{K}\right]$. To check this claim we plot in Fig. 7 the relative error of the perturbative RG method (compared to NRG) multiplied by $\ln \left[\sqrt{\omega^{2}+B^{2}} / T_{K}\right]$ for a wide range of $\omega$ and $B$. As expected, one obtains a number of order unity! Fig. 7 suggests that the next-order correction is suppressed by a factor smaller than $1.5 / \ln \left[\max (B, \omega) / T_{K}\right]$. From the large- $B$ expansion of quantities like the magnetization calculated by Bethe ansatz ${ }^{4,28}$ one

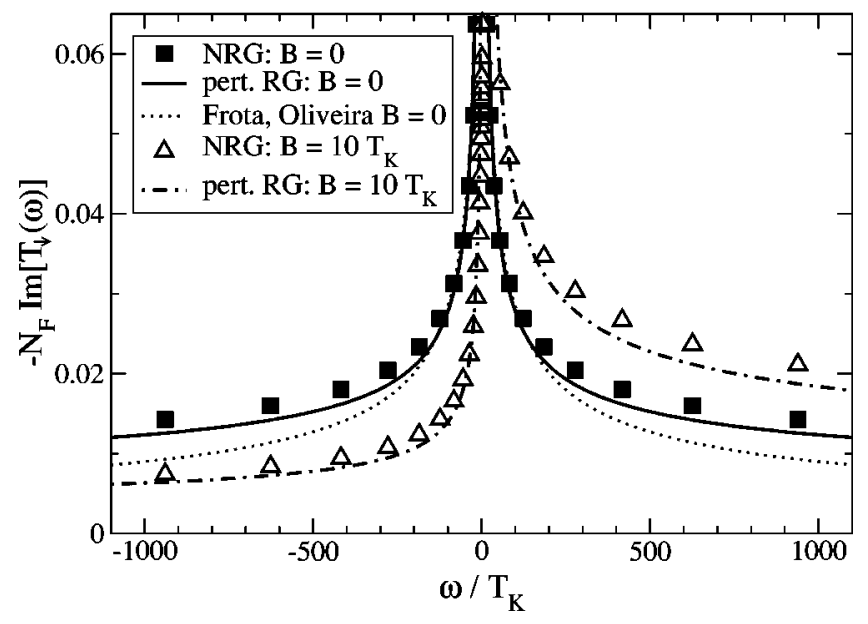

FIG. 6. Behavior of the spectral function $-N_{F} \operatorname{Im}\left[T_{\downarrow}(\omega)\right]$ $=-N_{F} V^{2} \operatorname{Im} G_{\downarrow}^{f}(\omega)$ for large $\omega$ and $B=0,10 T_{K}$. For $B, T_{K} \ll \omega$ the spectral function decays as $1 / \ln ^{2}\left[\omega / T_{K}\right]$ with prefactors depending on the magnetization [see Eq. (11)]. Symbols: NRG, solid and dot-dashed line: perturbative RG. For very large $\omega>200 T_{K}$ one can see that an interpolation formula suggested in Ref. 17 (dotted line) decaying as $1 / \sqrt{\omega}$ is not consistent (Ref. 29) with the NRG data.

expects that the correction rather has the form $\ln \left[\ln \left[\max (B, \omega) / T_{K}\right]\right] / \ln \left[\max (B, \omega) / T_{K}\right]$ but our range of parameters is too small to extract a $\ln [\ln [\ldots]]$ prefactor.

In the remainder of this section, we briefly compare our results to other approaches. Many years ago, Frota and Oliveira ${ }^{17}$ described the spectral function for $B=0$ with the simple heuristic form $N_{F} T(\omega)=-1 / \operatorname{Re}\left[\pi\left(1-i \omega / \Gamma_{K}\right)^{1 / 2}\right]$, where $\Gamma_{K} \approx 0.4 T_{K}$ (in our units) was fitted to the width of the Kondo peak. This form was chosen to interpolate between the exact $\omega=0$ result and an asymptotic $1 / \sqrt{\omega}$ behav-

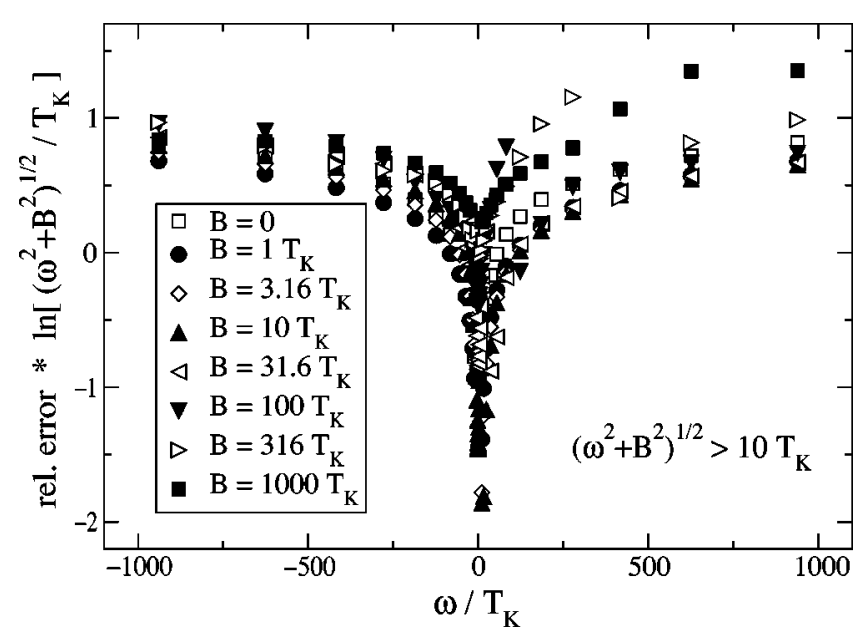

FIG. 7. The perturbative RG is a controlled expansion in the small parameter $1 / \ln \left[\max [\omega, B] / T_{K}\right]$. This is shown by plotting the relative error of the perturbative RG compared to NRG, (NRG-pert.RG)/NRG, multiplied by $\ln \left[\sqrt{\omega^{2}+B^{2}} / T_{K}\right]$ for various frequencies and magnetic fields. The perturbative $R G$ results have been broadened by the NRG resolution (see Fig. 3 and text). Only data points with $\sqrt{\omega^{2}+B^{2}}>10 T_{K}$ are shown. 
ior which was believed to arise as a consequence of an x-ray singularity in the presence of a phase-shift $\pi / 2$. The fit to the Frota and Oliveira formula works surprisingly well for frequencies up to $200 T_{K}$ (see Fig. 6). From our point of view, this remarkable agreement is, however, accidental in the following sense: For $T_{K} \ll \omega \ll D$ the spectral function decays as $1 / \ln ^{2}\left[\omega / T_{K}\right]$ and not as $1 / \sqrt{\omega}$ as has previously been pointed out by Dickens and Logan. ${ }^{29}$ Does a $1 / \sqrt{\omega}$ law hold at intermediate frequencies, e.g., for $T_{K} \ll \omega \ll T^{*}$ ? This would require the existence of a new scale $T^{*}$ parametrically larger than $T_{K}$, in contradiction not only to the perturbative RG but also to the exact Bethe Ansatz solution. ${ }^{28}$ Nevertheless, the Frota-Oliveira formula is a very good heuristic description in a large regime. For $20 T_{K}<\omega<200 T_{K}$ it is almost indistinguishable from our perturbative RG result for $B=0$ (and fits the exact result much better for $\omega<20 T_{K}$ where our perturbative scheme breaks down).

Recently, Logan and Dickens ${ }^{13,29}$ calculated the spectral functions in a magnetic field within their "local moment approach" (LMA), which is based on a combination of diagrammatic perturbation theory and mean-field theory. Interestingly, Logan and Dickens obtain the correct $\omega \rightarrow \infty$ and $\omega \rightarrow 0$ asymptotics. However the peak in the spectral function close to $\omega=B$ appears to be much broader in their approach and they find that the peak is shifted towards larger frequencies of order $B \ln \left(B / T_{K}\right)$. We think that both features are an artifact of their heuristic scheme to introduce broadening.

Moore and Wen ${ }^{12}$ calculated the density of states of the spinons obtained from the Bethe ansatz solution. ${ }^{28}$ They suggested that their result may serve as an approximation to the spectral function. However, the spinon density of states lacks the logarithmic tails characterizing the large $\omega$ behavior of the spectral function and their peaks close to $\omega=B$ do not show the correct asymmetric line shape for $B \gg T_{K}$. Nevertheless, it is interesting to note that the width of the spinon density of states is of order $\sim B / \ln ^{2}\left[B / T_{K}\right]$, like the left flank of our result. In their result, the position of the peak in the spectral function is shifted by $-B /\left(2 \ln \left[B / T_{K}\right]\right)$ away from $\omega=B$ for $B \gg T_{K}$. As discussed above, such a shift is beyond the precision of our present calculation but an interesting open question is whether the peak position in the spinon density of states coincides with that in the electron spectral function.

\section{CONCLUSION}

In this paper we have addressed three problems in particular.

(i) We have calculated analytically the behavior of the universal spectral function in the Kondo model for either large frequencies or large magnetic field and arbitrary frequencies. We hope that our results establishes the existence and detailed shape of the highly asymmetric peak at frequencies of the order of the Zeeman splitting and the asymptotic behavior at high frequencies. Hopefully, the spectral function in a magnetic field can be studied in some detail in future experiments, e.g., by tunneling into magnetic impurities.

(ii) In a detailed comparison with perturbation theory and NRG, we have argued that our recently developed perturbative RG scheme ${ }^{20}$ allows controlled calculations in the small parameter $1 / \ln \left[\max (\omega, B) / T_{K}\right]$. We expect that a similar statement holds out of equilibrium for large bias voltages $V$ $\gg T_{K}$ where a comparison with numerically exact results is not yet possible.

(iii) We have tried to assess the ability of the NRG method to resolve structures at high frequencies. As is obvious from the formulation of NRG, structures which are much sharper than their typical frequency are not correctly reproduced by the NRG scheme. However, our results suggest that the error induced by the logarithmic discretization mainly results in a simple broadening. The spectral function for large $B$ can serve as a test for future improvements of the NRG algorithms adapted to describe high- $\omega$ features more precisely, which may be useful, for example, in implementations of dynamical mean field theory using the NRG scheme. $^{36}$

\section{ACKNOWLEDGMENTS}

We thank J. Kroha, D.E. Logan, J. Martinek, and M. Vojta for helpful discussions and especially L. Glazman, who motivated us to check the validity of our RG approach in more detail. This work was supported in part by the CFN, the Emmy Noether program (A.R.) of the DFG, and the SFB 195 of the DFG (TAC). We also thank the Ørsted Laboratory for hospitality during parts of this work (J.P.).
${ }^{1}$ J. Li, W.-D. Schneider, R. Berndt, and B. Delley, Phys. Rev. Lett. 80, 2893 (1998); V. Madhavan, W. Chen, T. Jamneala, M. F. Crommie, and N. S. Wingreen, Science 280, 567 (1998); H. C. Manoharan, C. P. Lutz, and D. W. Eigler, Nature (London) 403, 512 (2000); N. Knorr, M. A. Schneider, L. Diekhöner, P. Wahl, and K. Kern, Phys. Rev. Lett. 88, 096804 (2002).

${ }^{2}$ D. Goldhaber-Gordon, H. Shtrikman, D. Mahalu, D. AbuschMagder, U. Meirav, and M. A. Kastner, Nature (London) 391, 156 (1998); S. M. Cronenwett, T. H. Oosterkamp, and L. P. Kouwenhoven, Science 281, 540 (1998); W. G. van der Wiel, S.
De Franceschi, T. Fujisawa, J. M. Elzerman, S. Tarucha, and L. P. Kouwenhoven, ibid. 289, 2105 (2000); J. Nygård, D. H. Cobden, and P. E. Lindelof, Nature (London) 408, 342 (2000).

${ }^{3}$ S. De Franceschi, R. Hanson, W. G. van der Wiel, J. M. Elzerman, J. J. Wijpkema, T. Fujisawa, S. Tarucha, and L. P. Kouwenhoven, Phys. Rev. Lett. 89, 156801 (2002).

${ }^{4}$ A. C. Hewson, The Kondo Problem to Heavy Fermions (Cambridge University Press, 1993).

${ }^{5}$ J. Kroha, Solid State Phys. 40, 216 (2000); A. Kaminski, and L. I. Glazman, Phys. Rev. Lett. 86, 2400 (2001); G. Göppert and H. Grabert, Phys. Rev. B 64, 033301 (2001); J. Kroha and A. Zawa- 
dowski, Phys. Rev. Lett. 88, 176803 (2002).

${ }^{6}$ W. Metzner and D. Vollhardt, Phys. Rev. Lett. 62, 324 (1989); A. Georges, G. Kotliar, W. Krauth, and M. J. Rozenberg, Rev. Mod. Phys. 68, 13 (1996).

${ }^{7}$ Y. Meir, N. S. Wingreen, and P. A. Lee, Phys. Rev. Lett. 70, 2601 (1993).

${ }^{8}$ O. Sakai, S. Suzuki, W. Izumida, and A. Oguri, J. Phys. Soc. Jpn. 68, 1640 (1999).

${ }^{9}$ O. Takagi and T. Saso, J. Phys. Soc. Jpn. 68, 1997 (1999).

${ }^{10}$ T. A. Costi, Phys. Rev. Lett. 85, 1504 (2000).

${ }^{11}$ W. Hofstetter, Phys. Rev. Lett. 85, 1508 (2000).

${ }^{12}$ J. E. Moore and X. G. Wen, Phys. Rev. Lett. 85, 1722 (2000).

${ }^{13}$ D. E. Logan and N. L. Dickens, Europhys. Lett. 54, 227 (2001), D. E. Logan and N. L. Dickens, J. Phys.: Condens. Matter 13, 9713 (2001).

${ }^{14}$ A. C. Hewson, J. Phys.: Condens. Matter 13, 10011 (2001).

${ }^{15}$ J. Martinek, Y. Utsumi, H. Imamura, J. Barnas, S. Maekawa, J. König, and G. Schön, cond-mat/0210006 (unpublished); J. Martinek, M. Sindel, L. Borda, J. Barnaás, J. König, G. Schön, and J. von Delft, cond-mat/0304385 (unpublished).

${ }^{16}$ K. G. Wilson, Rev. Mod. Phys. 47, 773 (1975); H. R. Krishnamurthy, J. W. Wilkins, and K. G. Wilson, Phys. Rev. B 21, 1003 (1980)

${ }^{17}$ H. O. Frota and L. N. Oliveira, Phys. Rev. B 33, R7871 (1986).

${ }^{18}$ O. Sakai, Y. Shimizu, and T. Kasuya, J. Phys. Soc. Jpn. 58, 3666 (1989).

${ }^{19}$ T. A. Costi, A. C. Hewson, and V. Zlatić, J. Phys.: Condens. Matter 6, 2519 (1994).

${ }^{20}$ A. Rosch, J. Paaske, J. Kroha, and P. Wölfle, Phys. Rev. Lett. 90, 076804 (2003).

${ }^{21}$ Adding a magnetic field also to the conduction electrons will not change the results in the scaling limit as the density of states of the up and down electrons at the Fermi energy remains independent of $B$.

${ }^{22}$ A. Rosch, J. Paaske, J. Kroha, and P. Wölfle (unpublished).

${ }^{23}$ The vertices are defined using a pseudospin representation of the spin, see Ref. 20.

${ }^{24}$ P. W. Anderson, J. Phys. C 3, 2436 (1970).
${ }^{25}$ A. Rosch, J. Kroha, and P. Wölfle, Phys. Rev. Lett. 87, 156802 (2001).

${ }^{26}$ While we are considering only equilibrium quantities, the proper replacements of bare and renormalized quantities is most easily done in the Keldysh formulation where it is easy to identify correctly the impurity magnetization in Eq. (11). In an equilibrium technique it would be far from obvious that $\tanh (B / 2 T)$ has to be replaced by $M$.

${ }^{27}$ D. C. Langreth, Phys. Rev. 150, 516 (1966); P. Nozières, J. Low Temp. Phys. 17, 31 (1974).

${ }^{28}$ N. Andrei, Phys. Rev. Lett. 45, 379 (1980); N. Andrei, Phys. Lett. 87A, 299 (1982).

${ }^{29}$ N. L. Dickens and D. E. Logan, J. Phys.: Condens. Matter 13, 4505 (2001).

${ }^{30}$ To extract a power law $x^{\alpha}=e^{\alpha \ln x}$ the variations of the argument of the $e$ function should be large compared to 1 .

${ }^{31}$ M. Salmhofer and C. Honerkamp, Prog. Theor. Phys. 105, 1 (2001).

${ }^{32}$ T. A. Costi, Proceedings of NATO ARW on "Concepts in Electron Correlations," Hvar, Croatia, edited by A. C. Hewson and V. Zlatić (Plenum Press, New York, 2003).

${ }^{33}$ For our NRG results we use the asymptotically exact two-loop definition $T_{K}=D_{0} \sqrt{2 N_{F} J} e^{-1 /\left(2 N_{F} J\right)}$ of the Kondo temperature to be identified with $T_{K}=D_{0} e^{-1 /\left(2 N_{F} J\right)}$ within our one-loop perturbative RG.

${ }^{34}$ T. A. Costi, in Density Matrix Renormalization, edited by I. Peschel, X. Wang, M. Kaulke, and K. Hallberg (Springer, Berlin, 1999).

${ }^{35}$ As long as a logarithmic discretization of energies is used within the NRG method, the use of a different broadening scheme will not improve the agreement significantly. However, in some specific cases, an improvement in resolving finite energy peaks within NRG can be obtained by making use of the self-energy as described in: R. Bulla, A. C. Hewson, and Th. Pruschke, J. Phys.: Condens. Matter 10, 8365 (1998).

${ }^{36}$ R. Bulla, Phys. Rev. Lett. 83, 136 (1999); R. Bulla, T. A. Costi, and D. Vollhardt, Phys. Rev. B 64, 045103 (2001); R. Zitzler, Th. Pruschke, and R. Bulla, Eur. Phys. J.B 27, 473 (2002); T. A. Costi and N. Manini, J. Low Temp. Phys. 126, 835 (2002). 